\title{
DAMAGE DETECTION IN A COMPOSITE BEAM WITH A DOUBLE EDGE CRACK USING FINITE ELEMENT ANALYSIS
}

\author{
Ramanamurthy E.V.V' Chandrasekaran $\mathrm{K}^{2}$ \\ ${ }^{1}$ Research Scholar, Jawarhalal Nehru Technological University, Andrapradesh, India. \\ ${ }^{2}$ Department of Mechanical Engineering, R.M.K.Engineering College, Chennai, India \\ E-mail: 1 evvrm.mech@gmail.com
}

\begin{abstract}
Cracks in vibrating component can initiate catastrophic failures. The presences of cracks change the physical characteristics of a structure which in turn alter its dynamic response characteristics. Therefore there is need to understand dynamics of cracked structures. Crack depth and location are the main parameters for the vibration analysis. So it becomes very important to monitor the changes in the response parameters of the structure to access structural integrity, performance and safety. A number of analytical, numerical and experimental techniques are available for the study of damage identification in beams. The objective of this paper is to develop a damage detection method in a composite cantilever beam with a double edge crack has been studied using finite element method. The vibration-based damage detection methods are based on the fact that changes of physical properties (stiffness, mass and damping) due to damage will manifest themselves as changes in the structural modal parameters (natural frequencies, mode shapes and modal damping). The task is then to monitor the selected indicators derived from modal parameters to distinguish between undamaged and damaged states. In the present study, vibration analysis is carried out on a cantilever beam with two open transverse cracks, to study the response characteristics and damage identification is based on the damage index method. Suitable boundary conditions are used to find out natural frequency and mode shapes. The results obtained numerically are validated with the results obtained from the simulation. The simulations have done with the help of ANSYS software. The importance of using damage patterns from the damage index method, (which is based on changes in modal strain energies) is that only measured mode shapes are required in the damage identification. The results shows, the damage index based method is capable of identification of damage and severity of damage in a structure.
\end{abstract}

Keywords: Open transverse cracks; Modal parameters; Damage Index; Damage Indicator; Damage severity.

\section{INTRODUCTION}

Structural damage can be broadly defined as any geometrical deviation or material property change that may degrade the integrity of the structure or result in unintentional dynamic response of the structure. Damage may be formed at different stages of a structure's service life. Major concerns in the operation of in-service structures are the reliability of the structure and the cost associated with the maintenance program. Unpredictable occurrence of damage may cause catastrophic failure and hereby possesses a potential threat to human lives. The importance of damage detection has led to continuous efforts to develop more efficient and rigorous technologies. It is highly desirable to pursue effective engineering solutions to detect, locate, quantify and predict the damage situation at the earliest possible stage.

The most fundamental issue is the fact that damage typically is a local phenomenon and may not dramatically influence the global dynamic response of a structure. For example, it is a common view that the frequency reduction has significant limitations for damage indication although the sensitivity varies from one structure to another. Therefore, damage may be difficult to detect until it becomes significantly severe.

This challenge is further supplemented by many other practical issues associated with measurements and signal processing errors, environmental or boundary condition deviation and modelling inaccuracy, etc. It is of interest to note that damping is probably the most sensitive structural parameter but it is also the most difficult one to obtain accurately. On the other hand, natural frequencies are probably the most accurately achievable modal parameters but their sensitivity to localised damage may be very low. Accurate mode shapes, which are difficult to obtain, can be used for damage identification but such an approach requires high spatial resolution. A large number of transducers are needed to obtain meaningful mode shapes. Furthermore, the full-scale modal 
analysis is not an easy task, especially for large structures with high-density modes.Most of the work reported in literature is based on analytical simulations. Lack of experimental validation eventually degrades the credibility of the proposed method. Since measurements are always contaminated with noises, the outcomes from the field tests may be quite different to those derived from analytical simulations.

Used in conjunction with natural frequencies, mode shape approach has also received considerable attention in the literature. Deterioration of a structure can alter local stiffness and cause discontinuity near the region. The consequent change of mode shapes depends on both the severity and the location of the damage. The magnitude of change with respect to each mode may vary from one to another. This can be used as a tool to predict the location of the damage.

Another class of damage detection method is model-based approaches, typically relying on the Finite Element (FE) model updating techniques. The basic idea is to modify the objective parameters in a base FE model so that the model predictions match the measured data in an optimal way. Two steps are often involved in the damage detection process. The first step is to produce a validated finite element model of the undamaged state as a baseline. Then the model is updated to obtain a model in damaged state matching the measured vibration data at the damaged state. Comparisons of the updated matrices to the baseline model provide an indication of the damage and can be used to quantify the location and extent of damage. A large number of physical parameters are needed to define a typical FE Model while the experimental modal parameters used to guide the tuning up of the model are limited.

Due to its importance, damage detection has attracted considerable attention from the engineering research community in the past several decades. For structures that require high reliability such as aerospace vehicles, transportation systems, offshore platforms and various civil structures, etc., it is vital to maintain systems at an optimally healthy condition. The continuous demand for effective, fast and economical damage detection methods has led to the development of vibration-based approaches that examine changes in the vibration characteristics of the structure. Modal parameters such as frequencies, damping ratios and mode shapes are the main vibration characteristics considered in these approaches. During the last several decades, extensive analytical, numerical and experimental investigations have been conducted for various applications.

When a structure suffers from damages, its dynamic properties can change, especially, crack damage can cause a stiffness reduction, with an inherent reduction in natural frequencies, an increase in modal damping, and a change of the mode shapes. The review will mainly focus on vibration-based damage detection methods and summarize some remarkable points. Some representative works are cited and discussed here according to the category in which the damage indicator was used. Armon, et al. [1] used the idea of rank-ordering of the modes according to the fractional reduction of natural frequencies. It was shown both by simulation and experiment that the rank-ordering of the modes based on frequency shifts was useful for determining the crack location. The method claimed to be not sensitive to small uncertainties in the boundary conditions and changes in the surrounding temperature. Cawley and Adams [2] gave a formulation to detect damage in composite materials from frequency shifts. An error term was constructed that relates the measured frequency shifts to those predicted by a model based on a local stiffness reduction. Special considerations were given to the anisotropic behaviour of the composite materials. Cerri and Ruta [3]) investigated the detection of the structural damage affecting a narrow zone of a doubly hinged plane circular arch by means of a few measured natural frequencies. The localised damage was modelled as a torsion spring joining two adjacent sections and characterised by the location and the stiffness of the spring. Chondros and Dimarogonas [4] investigated the influence of a crack in welded joints. They modelled a cantilever beam with a mass at the free end and a crack at the welded root. The change of the bending stiffness due to the crack was measured experimentally and then used in the mathematical model for other boundary conditions. Chondros et al. [5] has analyzed the lateral vibration of cracked Euler-Bernoulli beams with single or double edge cracks. Their analysis can be used for the prediction of the dynamic response of a simply supported beam with open surface cracks. Dimarogonas and Massouros [6] studied the torsional vibration of a shaft with a circumferential crack and modelled the cracked section as a torsional spring. The stiffness change due to a 
crack was also modelled as reduction in the local elastic modulus by some researchers. Gounaris and Dimarogonas [7] investigated a cracked prismatic beam element for structural analysis. Similar to above discussed local flexibility matrix, the relationship between the displacement and force vectors was formulated with respect to the element containing the crack. One could then combine this flexibility matrix with any finite element program for further analysis. Gudmundson [8] presented a first order perturbation method which predicts changes in natural frequencies of a structure resulting from cracks, notches or other geometrical changes. The method was applied to an edge-cracked rectangular beam. Fine-mesh finite element techniques were used to obtain local flexibility by Gudmundson [ 9], and Krawczuk and Ostachowicz [10], etc. These contributions made it possible to investigate the dynamic behaviour of cracked structures in a systematic way. For more complex structures, finite element techniques are usually applied to analyse the dynamic response of the structure. Lifshitz and Rotem [11] proposed a damage detection method based on the change in the dynamic moduli, which could be related to the frequency shift. The dynamic moduli were the slopes of the extensional and rotational stress-strain curves under dynamic loading. Maia,et al. [12] suggested a new version of mode shape-based method by generalising them to the whole frequency ranges of measurement. A series of numerical simulations on a simple beam were made in order to compare various damage detection methods based on mode shape changes. The experimental results showed a certain degree of agreement. Pandey and Biswas [13] studied the flexibility approach based on the computation of changes in the flexibility matrix of the structure using experimentally obtained modal parameters. It was shown that the flexibility of a structure converged rapidly with increasing frequency and a good estimate of the flexibility matrix was obtained from just the first two modes. A beam under three types of boundary conditions was investigated analytically and experimentally. Both results showed that the flexibility was fairly sensitive to the amount of damage. The author stated that the proposed method worked best when damage is located at a section where high bending moments occur.

Pandey, et al. [14] introduced the curvature mode shapes as a possible candidate to detect and locate damage in a structure. By using a cantilever and a simply supported analytical beam model, it was demonstrated that the absolute changes in mode shape curvature were localised in the region of damage and hence can be a good indicator of damage in the structure. Finite element analysis was used to obtain the displacement mode shapes of the two models. The curvature values were computed from the displacement mode shapes using a central difference approximation. Patil and Maiti [15] have utilized a method for prediction of location and size of multiple cracks based on measurement of natural frequencies has been verified experimentally for slender cantilever beams with two and three normal edge cracks. The analysis is based on energy method and representation of a crack by a rotational spring. For theoretical prediction the beam is divided into a number of segments and each segment is considered to be associated with a damage index. The damage index is an indicator of the extent of strain energy stored in the rotational spring. The crack size is computed using a standard relation between stiffness and crack size. Number of measured frequencies equal to twice the number of cracks is adequate for the prediction of location and size of all the cracks.

Rizos, et al. [16] developed a method of measuring the amplitude of a steel beam at two points during forced vibration at one of its natural frequencies. Analytical results were used to relate the measured vibration modes to the crack length and depth. The identification method was based on the assumption of a transverse surface crack, extending uniformly along the width of the structure. Mode shapes were measured by using two calibrated accelerometers mounted on the beam. An intensive review of literature on this category was made by Salawu [17]. The most appealing feature associated with using natural frequencies is that natural frequencies are relatively cheap to obtain and easy to extract. Stubbs and Kim (18) stated that, the damage identification based on the changes in the curvature of the $j_{\text {th }}$ mode at location $i$ and formulated an damage index parameter.

However, there are some drawbacks on using mode shape methods. First, it is not an easy task to obtain the meaningful mode shapes, especially for large structures. Confident identification of the damage requires proper number of sensors and right choice of sensor coordinates. Another concern is that damage is a local phenomenon and may not significantly influence 
mode shapes of the lower modes that are usually measured from vibration tests of large structures.

There often exist situations where direct use of modal parameters is not sensitive to the local damage. Considerable efforts have been spent by researchers to seek alternative indicators to detect damage. Some attempts were using derived modal parameters, such as mode shape curvature and modal strain energy. The drive to use vibration-based methods for damage detection is the fact that the presence of a crack or localised damage in a structure results in the stiffness reduction, mass loss and damping increase, which affects the dynamic characteristics of the structure. How to quantify such effects is an important aspect of the research for the successful implementation of these methods.

There are constant demands for the investigation of more effective damage detection techniques. The sensitivity problem is the most common obstacles for the success of the techniques. Another issue is the dependence on a very accurate analytical model. Obtaining an accurate model in itself is not an easy task. Even when the analytical model is present, to predict and locate the damage, these methods require excessive post-processing after data collection.

Graphical comparisons of relative changes in mode shapes were claimed to be a better way to locate the damage when only resonant frequencies and mode shapes were examined. In general, the changes in mode shape seem to become more pronounced as the mode number increases.To investigate the alternative methods, it may be possible to study the local member instead of the whole structure and observe the change of the behaviour rather than just quantitative change of the parameters. This philosophy is the motivation for the current studies.

\section{THE FINITE ELEMENT ANALYSIS}

The general purpose finite element computer software ANSYS was used to carry out the finite element analysis in the frequency domain and obtain natural frequencies, mode shapes and transfer functions. An eight node linear layered 3-D shell element with six degrees of freedom at each node (Specified as Shell 99 element in ANSYS) was selected based on convergence test and used throughout the study. Each node has six degrees of freedom, making a total forty eight degrees of freedom per element. The Figure (1) represents the detail and Geometry of element type. The figure (2) represents the layer stacking by using ANSYS

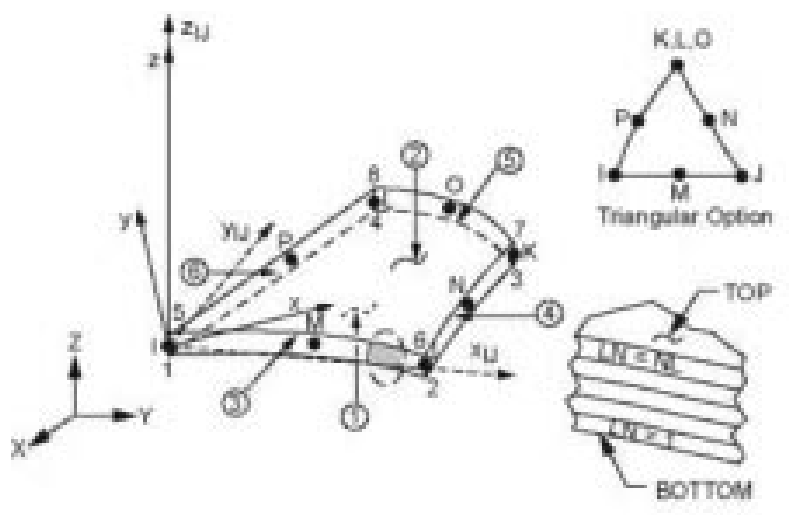

Fig 1. Details of Shell 99 Element

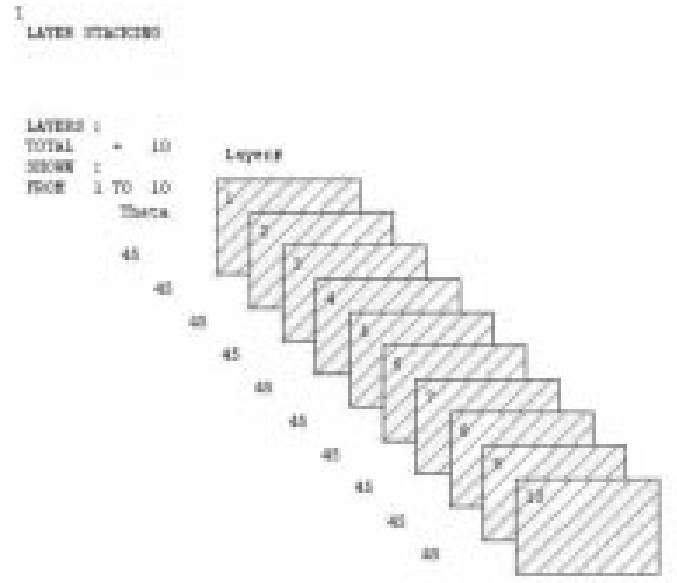

Fig. 2. Layer stacking in ANSYS

A Cantilever composite beam with a double edge crack was considered for the numerical studies by using ANSYS software. From the numerical analysis, first three bending modes were extracted the mode shape displacements are calculated at these frequencies. The analysis was carried out by considering only bending mode shapes.

Numerical modal analysis based on the finite element $(F E)$ modeling is performed for studying the dynamic response of a structure. The natural frequencies and mode shapes are important modal parameters in designing a structure under dynamic loading conditions. The numerical analysis is carried out by using the commercial finite element program ANSYS. It is mainly used to verify the effectiveness of damage detection algorithm used in this study. In the 
present study, since the specimen is made of fiberglass/epoxy composite material, the FE modeling of the composite beam is simulated with the layered element (Shell 99). The composite plate consists of several orthotropic layers, and it is considered as orthotropic material. The FE analysis software ANSYS was used in the modal analysis to obtain mode shapes.

A Cantilever beam of the dimensions L: W:t of composite material was considered for the numerical analysis. The beam was modeled with eight nodded shell elements so as to introduce the crack and analyze for the propagation of the crack. Only the growth of crack through the thickness is modeled and also, the crack opening and closing has not been explicitly considered in the analysis. Hence the process has been taken to be linear.

\section{EVALUATION OF DAMAGE DETECTION}

This detection technique is extended in the present study, and the amplitude difference of curvature mode shapes is employed to estimate the local stiffness loss due to damage. The first curvature mode is used in the damage assessment, since the curvature nodal points of damaged structures at high modes may shift significantly from the original undamaged case thus generating misleading results. It is relatively difficult to experimentally obtain the mode shapes at the higher modes using surface-bonded sensors; while at the lower modes, the mode shapes may not be sensitive to small damage. In this study, the first three low curvature mode shapes of the structures between an undamaged state and a damaged state are extracted from the finite element analysis data. Then, the differences of these curvature modes are used to determine damage locations and the corresponding magnitudes. The procedure illustrates the damage state from the original undamaged state; however, it can also be modified to consider the changes from one damaged state to another damaged state. The study is conducted by using Glass / Epoxy laminated composite beam, and the curvature mode shapes are measured by using FEM software ANSYS. The results of damage identification by using damage index method for the Glass / Epoxy laminated beam are presented.

A study is made to compare the change in frequencies, obtained due to the presence of a crack in a composite beam with an undamaged beam. The general purpose FEM analysis software ANSYS was used in the modal analysis module to obtain mode shapes. The damage case considered for this analysis:

DAMAGE CASE: Damage location at $50 \mathrm{~mm}$ and at $100 \mathrm{~mm}$ from the fixed end of composite cantilever beam.

The following Damage Index Method algorithm is used to locate the damage in the composite beam buy using FE analysis. The modal parameters that change locally are mode shapes, i.e., Bending mode shape The above methods is used in the evaluation of damage in the composite beam for bending shape. The results obtained were tabulated, plotted and discussed in the next section

\section{A. The Damage Index Method}

Stubbs and Kim (17) stated that, the damage identification based on the changes in the curvature of the $j$ th mode at location $i$. The damage parameter formulation of this method is presented as

$$
\frac{\left(\left\{\varphi_{d}^{\prime \prime}\right\}_{i, j}^{2}+\sum_{1}^{i \max }\left\{\varphi_{d}^{\prime \prime}\right\}_{i, j}^{2}\right) \cdot \sum_{1}^{i \max }\left\{\varphi_{d}^{\prime \prime}\right\}^{2}}{\left(\left\{\varphi_{d}^{\prime \prime}\right\}_{i, j}^{2}+\sum_{1}^{i \max }\left\{\varphi_{u d}{ }^{\prime \prime}\right\}_{i, j}\right) \cdot \sum_{1}^{i \max }\left\{\varphi_{d}^{\prime \prime}\right\}_{i, j}^{2}}
$$

where $\beta_{i j}$ is the damage index at location $i$ for mode $\left\{j, \varphi_{u d} d^{\prime \prime}\right.$ is the second derivative or curvature of mode shape $\varphi$ for undamaged beam, with respect to $x \cdot \varphi_{d}^{\prime}$ is the second derivative or curvature of mode shape $\varphi$ for damaged beam, with respect to $x$.

To establish a comparative basis for different modes, the damage index values is transformed into the normal standard normal space, and the normalized damage indicator $Z_{i j}$ is given by,

$$
Z_{i j}=\frac{\beta_{i j}-\mu_{\beta i j}}{\sigma_{\beta i j}}
$$

with $\mu_{\beta i j}$ the mean and $\sigma_{\beta i j}$ the standard deviation of the $\beta_{i j}$ values for all $j$ elements. The estimation of the damage severity for element $j$ is expressed $d$ by, 


$$
\alpha_{i j}=1-\frac{1}{\beta_{i j}}
$$

Where $\alpha_{i j}$ is damage severity estimator.

Positive $Z_{i j}$ and $\alpha_{i j}$ values indicate the possibility of damage and can therefore be utilized to locate and quantify defects, respectively.

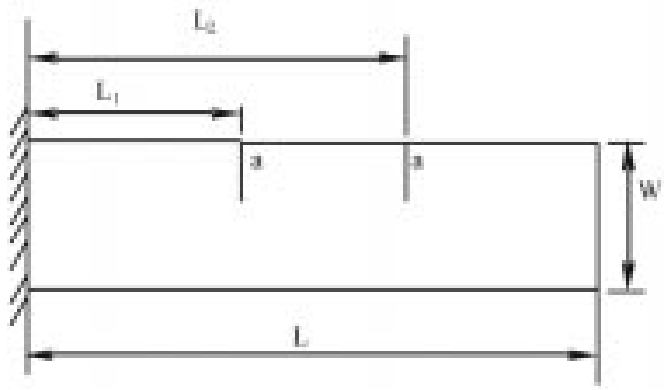

Fig 3. Geometry of cantilever beam with a double edge crack

Figure 3. represents the geometry of cantilever beam with a double edge crack and the dimensions are:

Length $=L=400 \mathrm{~mm} ;$ Width $=W=30 \mathrm{~mm}$;

Thickness $=t=5 \mathrm{~mm}$; Number of layers $=N=10$;

Fiber orientation $=\theta=45^{\circ}$

$L_{1}=$ First damage location from fixed end;

$L_{2}=$ Second damage location from fixed end;

$a=$ Depth of crack at $L_{1}$ and $L_{2}$ respectively (crack is considered as damage);

Properties of Glass-Epoxy composite material for the analysis:

Young's modulus of fiber $=E_{f}=72.4 \mathrm{GPa}$;

Young's modulus of matrix $E_{m}=3.45 \mathrm{GPa}$;

Modulus of rigidity of fiber $=G_{f}=29.67 \quad \mathrm{GPa}$;

Modulus of rigidity of matrix $G_{m}=1.277 \quad \mathrm{GPa}$;

Poisson's ratio $=\gamma_{f}=0.22$;
Poisson's ratio $=\gamma_{m}=0.35$;

Density of fiber $=\rho_{f}=2.6 \mathrm{gr} / \mathrm{cm}^{3}$;

Density of matrix $=\rho_{m}=2.6 \mathrm{gr} / \mathrm{cm}^{3}$;

To study the damage scenarios at different locations and of various intensities a total of three damages were investigated. These relate to damage at two locations each having three levels of severity. The following damage case was considered for the analysis:

\section{B. Damage Case}

Damage location at $50 \mathrm{~mm}$ and at $100 \mathrm{~mm}$ from the fixed end of composite cantilever beam.

The First three bending mode shapes were considered for the identification and the severity of the damage.

The three damage severities termed as:

(a): Light damage (3 mm crack depth)

(b): Medium damage (6 $\mathrm{mm}$ crack depth)

(c): Heavy damage (9 $\mathrm{mm}$ crack depth)

\section{RESULTS AND DISCUSSIONS}

In FEM analysis the first three bending modes were considered.

Damage location at $50 \mathrm{~mm}$ and at $100 \mathrm{~mm}$ from the fixed end of composite cantilever beam. The three damage severities termed as:

(a) Light damage (3 $\mathrm{mm}$ crack depth)

(b) Medium damage (6 $\mathrm{mm}$ crack depth)

(c) Heavy damage (9 $\mathrm{mm}$ crack depth)

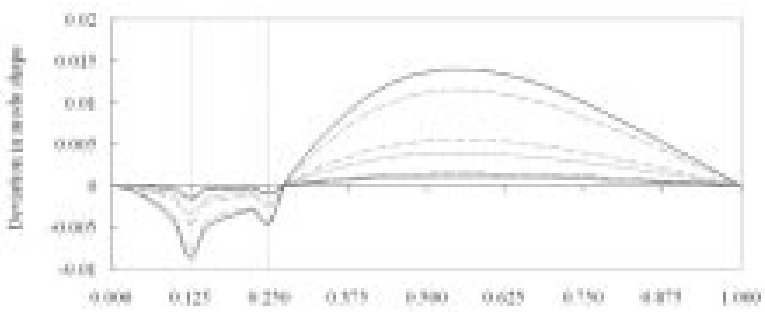

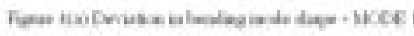




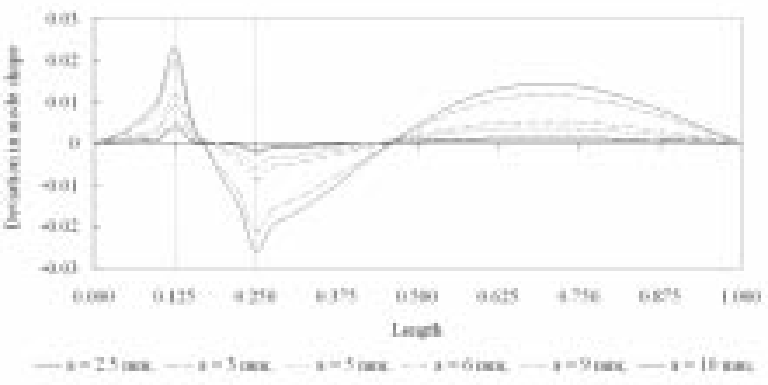

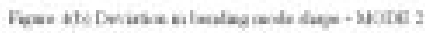

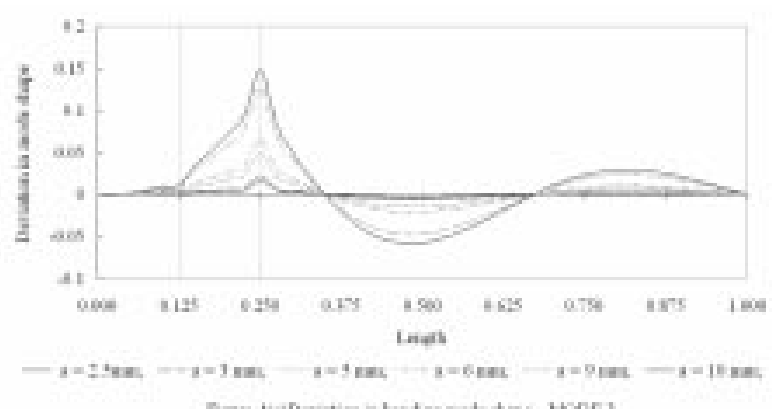

Figures 4(a) to 4(c) represents the deviation in mode shapes for different crack depths.

DAMAGE CASE : Damage location at $50 \mathrm{~mm}$ and at $100 \mathrm{~mm}$ from the fixed end of composite cantilever beam.
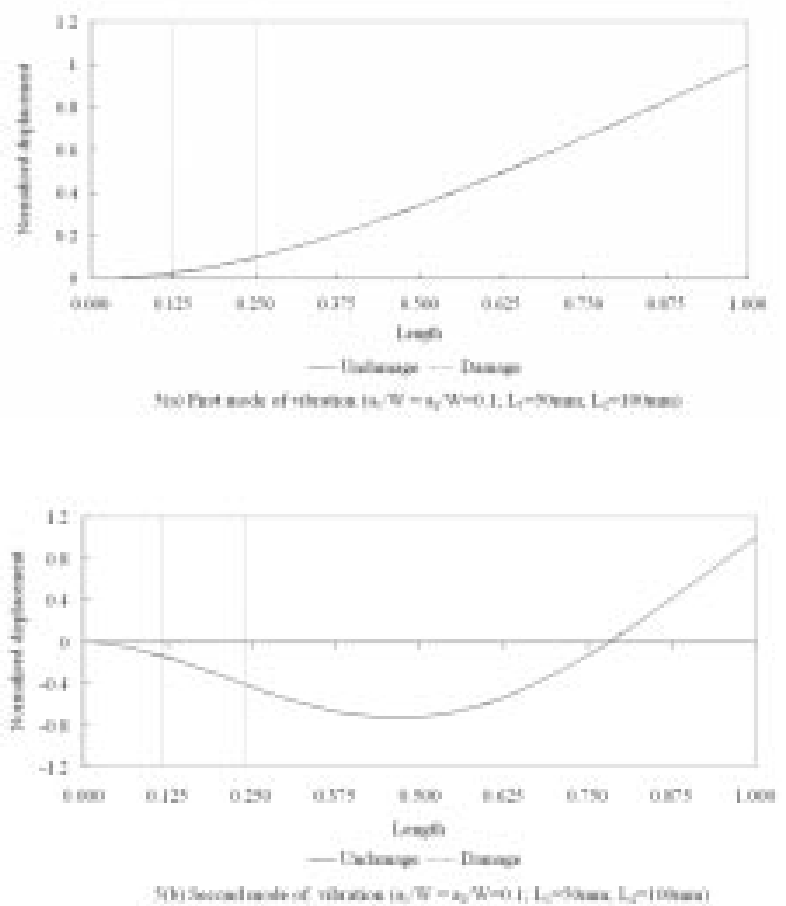

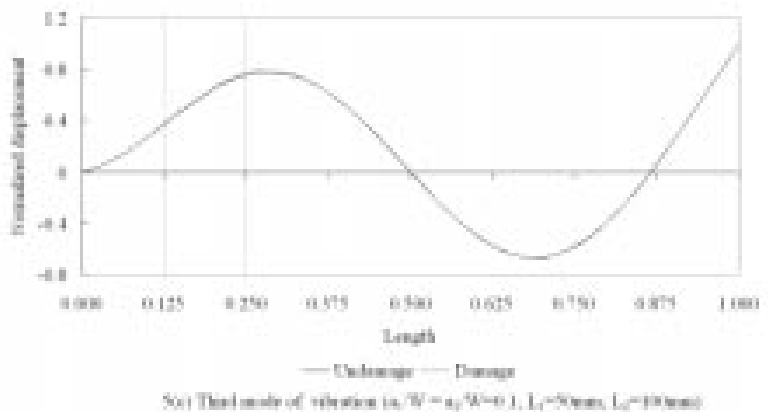

Fig.

(a) Light damage (3 $\mathrm{mm}$ crack depth) Figures 5(a) to5(c) represents normalized bending mode shapes
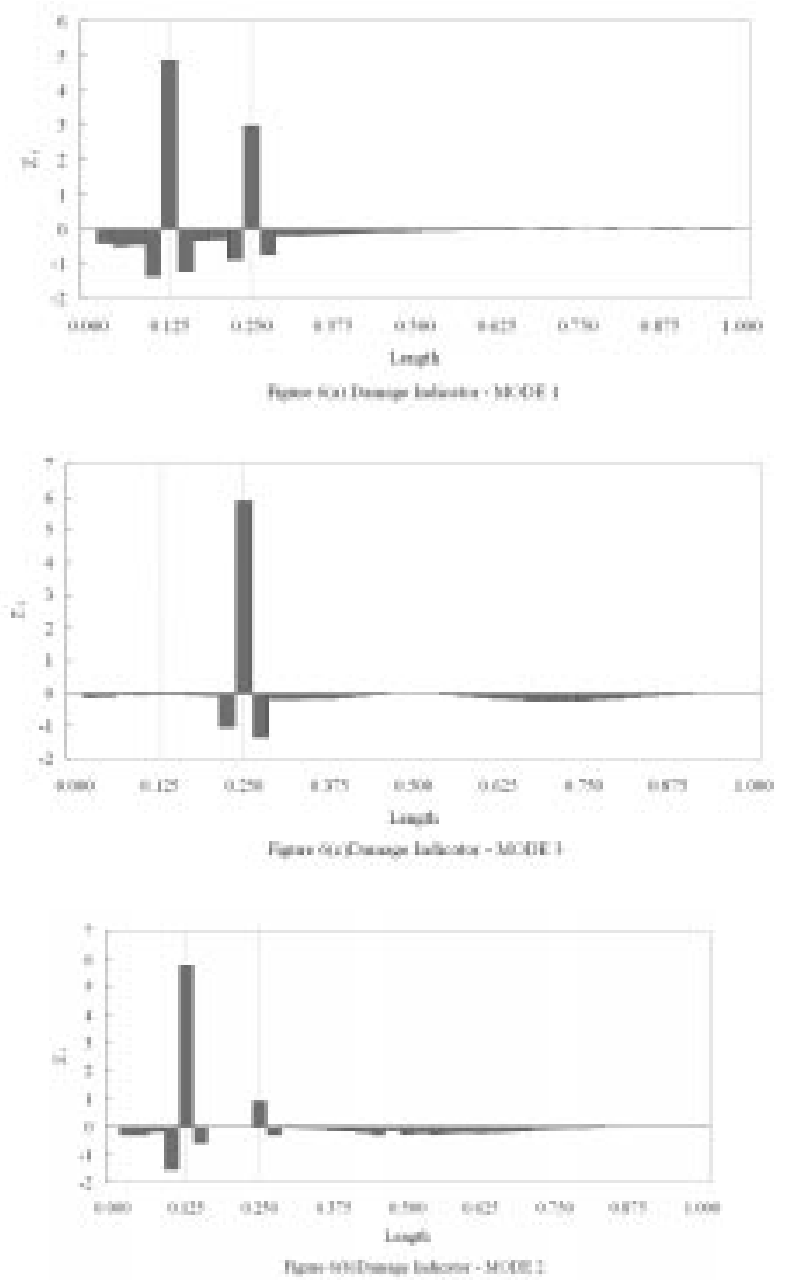

From figures 6(a) to 6(c) represents the damage indicator for (Light damage- $3 \mathrm{~mm}$ crack depth) first mode, second mode, and third mode respectively. 

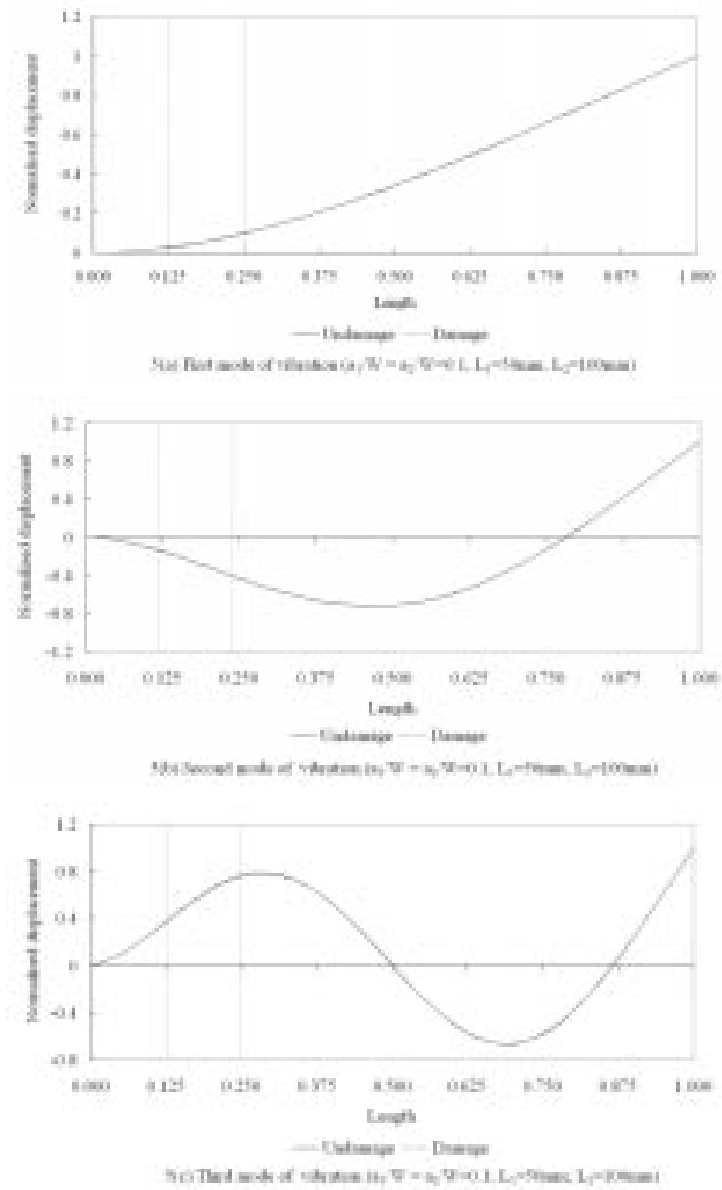

(b) Medium damage (6 $\mathrm{mm}$ crack depth)

Figures $7(\mathrm{a})$ to $7(\mathrm{c})$ represents normalized bending mode shapes
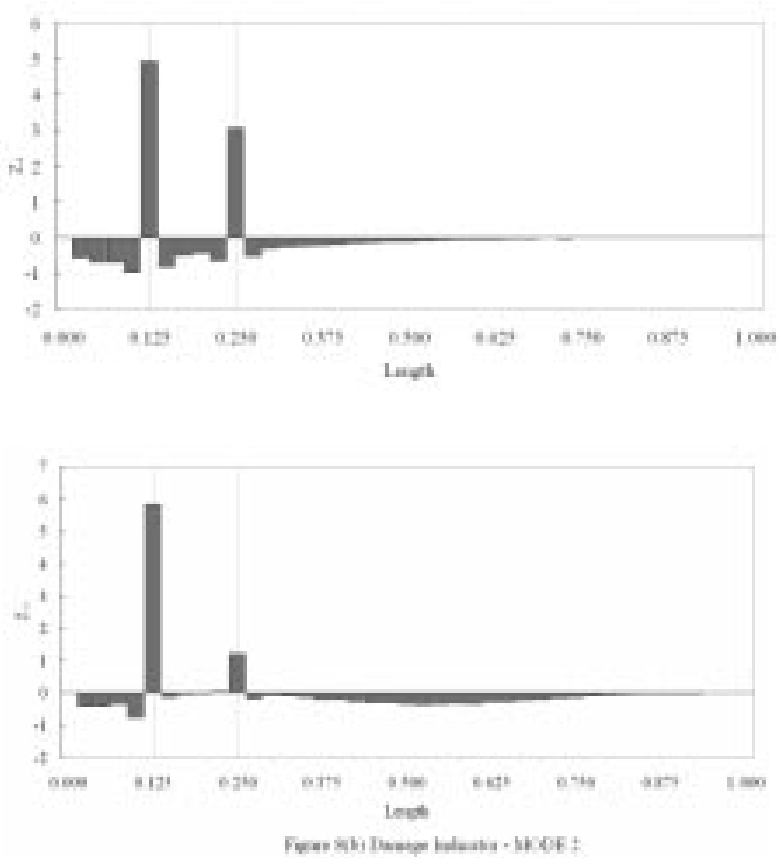

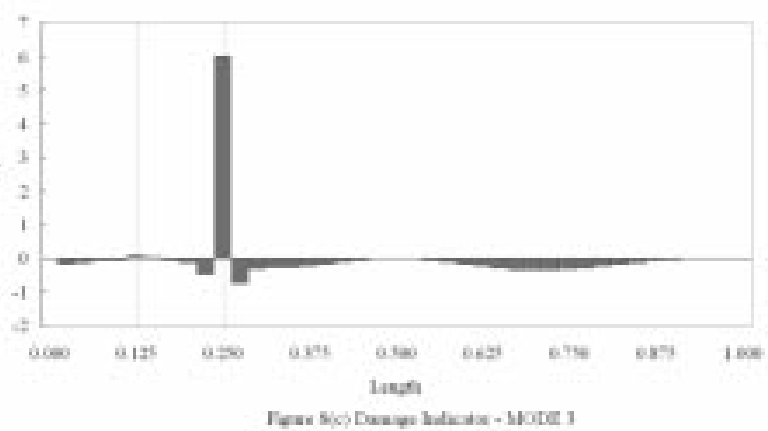

Figures $8(a)$ to $8(\mathrm{c})$ represents the damage indicator for (Medium damage- $6 \mathrm{~mm}$ crack depth) first mode, second mode, and third mode respectively.
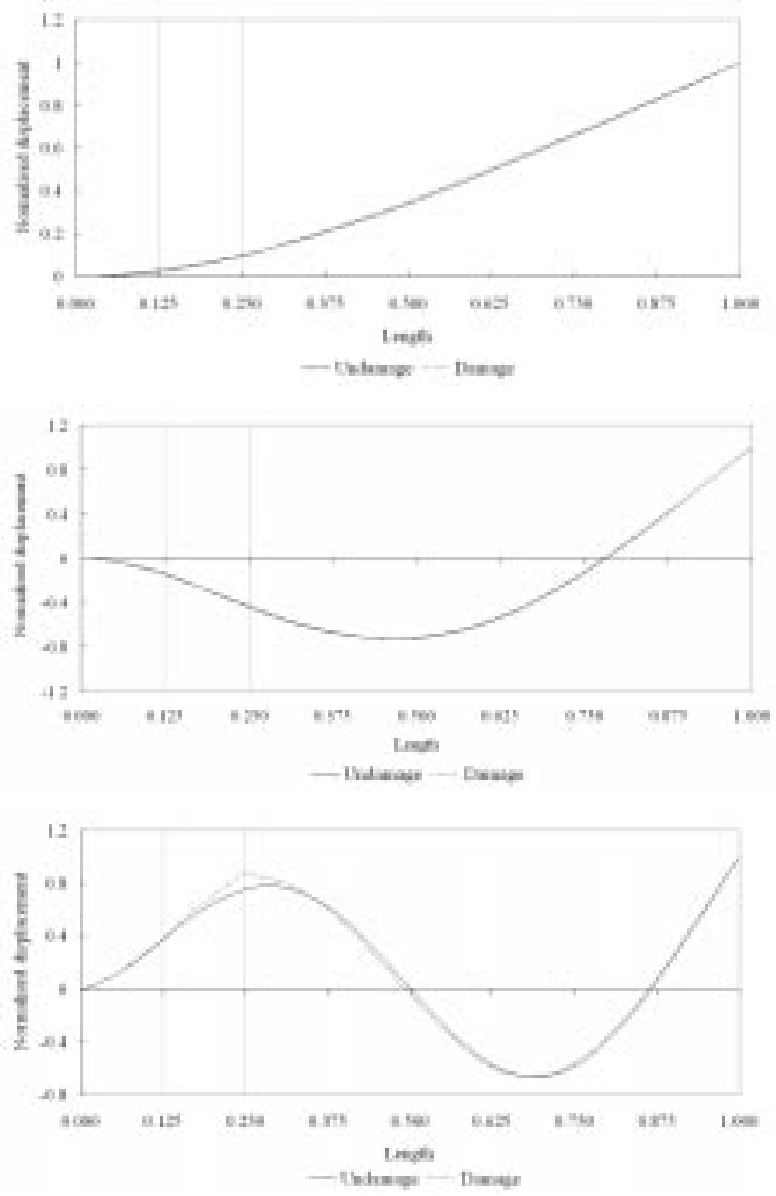

(c) Heavy damage (9 $\mathrm{mm}$ crack depth)

Figures $9(\mathrm{a})$ to $9(\mathrm{c})$ represents normalized bending mode shapes

From figures $10(a)$ to $10(\mathrm{c})$ represents the damage indicator for (Heavy damage- $6 \mathrm{~mm}$ crack depth) first mode, second mode, and third mode respectively. 

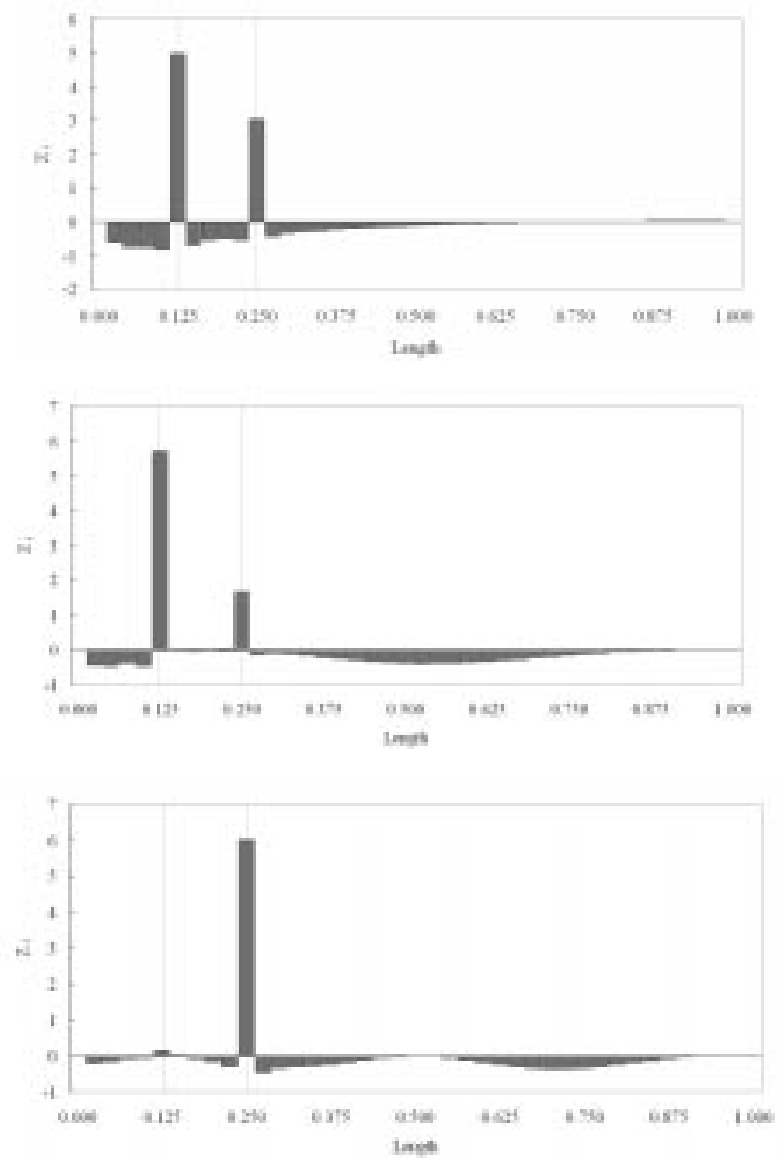

DAMAGE SEVERITY

Damage location at $50 \mathrm{~mm}$ and at $100 \mathrm{~mm}$ from the fixed end of composite cantilever beam.
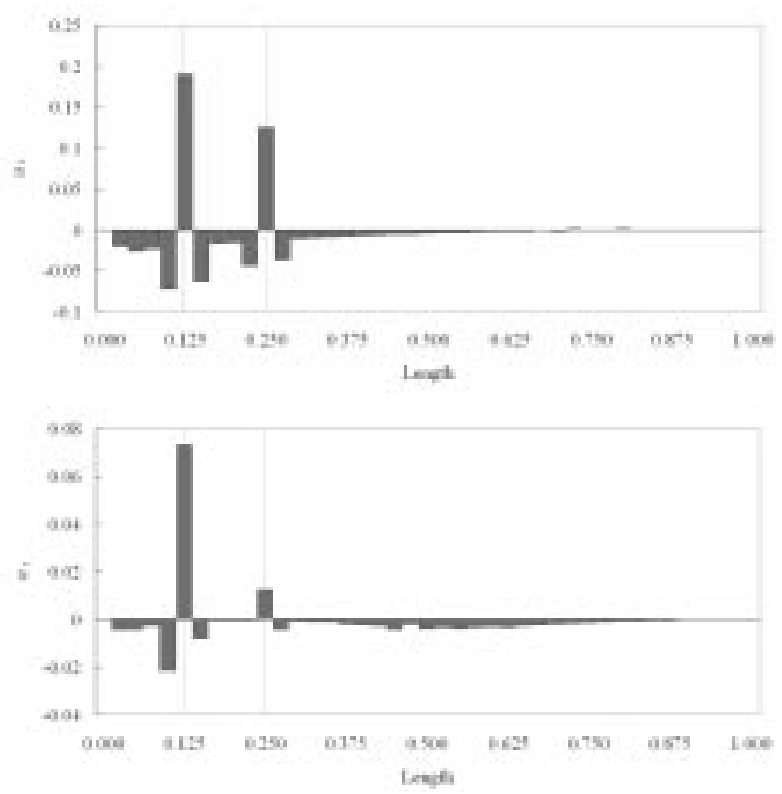

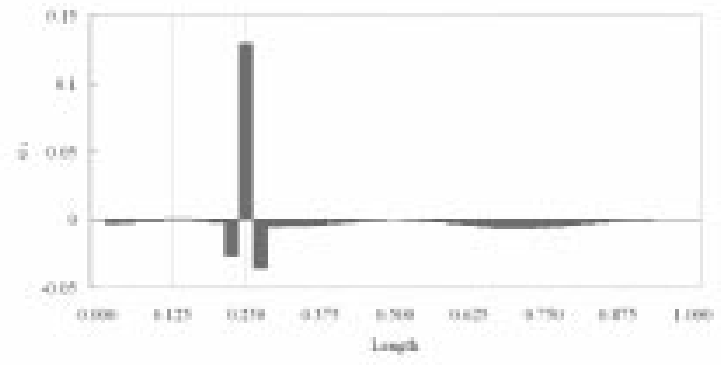

Figures 11(a) to 11(c) represents the damage indicator for (Light damage- $3 \mathrm{~mm}$ crack depth) first mode, second mode, and third mode respectively.
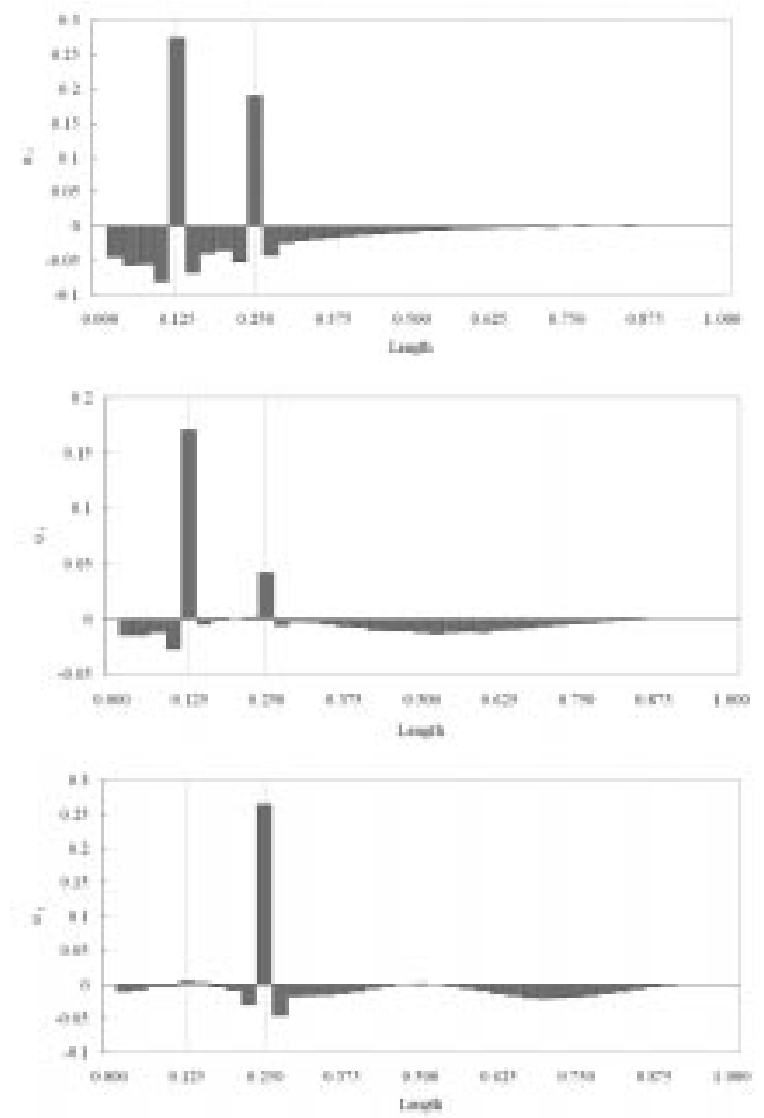

Figures 12(a) to 12(c) represents the damage indicator for (Medium damage- $6 \mathrm{~mm}$ crack depth) first mode, second mode, and third mode respectively.

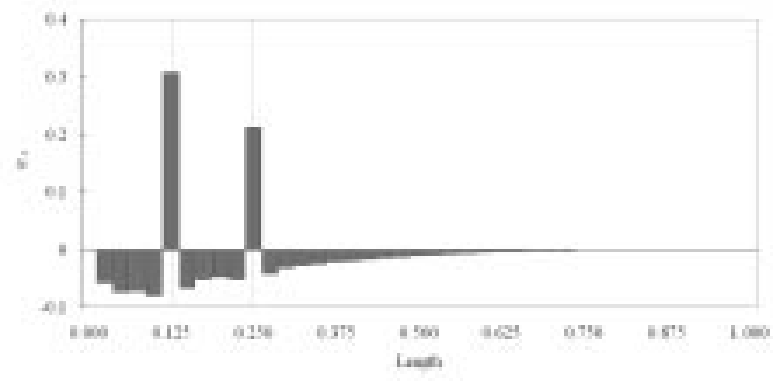




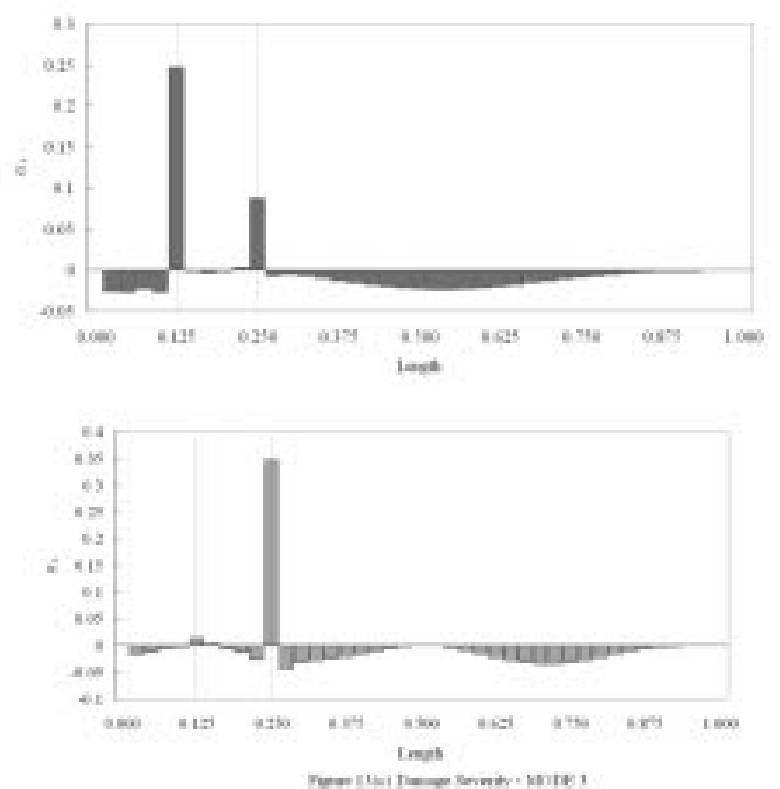

Figures $13(\mathrm{a})$ to $13(\mathrm{c})$ represents the damage indicator for (Heavy damage- $9 \mathrm{~mm}$ crack depth) first mode, second mode, and third mode respectively.

\section{CONCLUSION}

The proposed damage identification method utilizes damage indices from the damage index method, which is based on mod shape curvature changes. By analyzing the curvature of mode shapes the observed local mode shape changes are further enhanced giving better and more robust damage identification results. Positive $Z_{i j}$ and $\alpha_{i j}$ values indicate the possibility of damage and can be utilize to locate and quantity defects respectively. The actual damage location is marked with a straight line. The actual damage locations are marked with a dotted line.

The figures $6(\mathrm{a}), 6(\mathrm{~b}), 6(\mathrm{c})$ for light damage; $8(\mathrm{a})$, 8(b), 8(c) for medium damage; and 10(a), 10(b), 10(c) for heavy damage; The positive values of $Z_{i}$ indicates the damage factor of First mode, Second mode and Third mode respectively of the beam with damage locations at $50 \mathrm{~mm}$ and $100 \mathrm{~mm}$ from fixed end. Here, a clear indication of the damage location is visible in first mode and second mode of vibration.

The figures 11(a), 11(b), 11(c) for light damage; 12(a), 12(b), 12(c) for medium damage; and 13(a), 13(b), 13(c) for heavy damage; The positive values of $\alpha_{i j}$ indicates the damage severities values of First mode, Second mode and Third mode respectively of the beam with damage locations at $50 \mathrm{~mm}$ and 100 $\mathrm{mm}$ from fixed end. Here, a clear indication of the damage severity is visible in first mode and second mode of vibration.

The varying magnitude of $a_{i}$ clearly indicates different damage extents.

From the above results, it can be conclude that, damage identification method used in this study is satisfactory for damage identification and damage severity in all three cases.

\section{REFERENCES}

[1] Armon, D., Benhaim, Y. and Braun, S. (1994). "Crack detection in beams by rank-ordering of eigenfrequency shifts", Mechanical Systems and Signal Processing, Vol. 8, pp. 81-91.

[2] Cawley, P. and Adams, R. D. (1979). "Location of defects in structures from measurements natural frequencies", Journal of Strain Analysis for Engineering Design, Vol. 14, pp. 49-57.

[3] Cerri, M. N. and Ruta, G. C. (2004). "Detection of localised damage in plane circular arches by frequency data", Journal of Sound and Vibration, Vol. 270, pp. 39-59.

[4] Chondros, T. G. and Dimarogonas, A. D. (1980). "Identification of cracks in welded-joints of complex structures", Journal of Sound and Vibration, Vol. 69, pp. 531-538.

[5] Chondros T.G, Dimarogonas A.D and Yao, J. A. continuos cracked beam vibration theory, Journal of Sound and Vibration, 215, (1998), pp 17-34

[6] Dimarogonas, A. and Massouros, G. (1981). "Torsional vibration of a shaft with a circumferential crack", Engineering Fracture Mechanics, Vol. 15, pp. 439-444.

[7] Gounaris, G. and Dimarogonas, A. (1988). "A finite-element of a cracked prismatic beam for structural-analysis", Computers \& Structures, Vol. 28, pp.309-313.

[8] Gudmundson, P. (1982). "Eigen frequency changes of structures due to cracks, notches or other geometrical changes", Journal of the Mechanics and Physics of Solids, Vol. 30, pp. 339-353.

[9] Gudmundson, P. (1983). "The dynamic behavior of slender structures with cross-sectional cracks", Journal of the Mechanics and Physics of Solids, Vol. 31, pp. 329-345.

[10] Krawczuk, M. and Ostachowicz, W. M. (1993). "Transverse natural vibrations of a cracked beam loaded with a constant axial force", Journal of Vibration 
and Acoustics-Transactions of the Asme, Vol. 115, pp. 524-528.

[11] Lifshitz, J. M. and Rotem, A. (1969). "Determination of reinforcement unbonding of composites by a vibration technique", Journal of Composite Materials, Vol. 3, pp. 412-423.

[12] Maia, N. M. M., Silva, J. M. M., Almas, E. A. M. and Sampaio, R. P. C. (2003). "Damage detection in structures: From mode shape to frequency response function methods", Mechanical Systems and Signal Processing, Vol. 17, pp.489-498.

[13] Pandey, A. K. and Biswas, M. (1994). "Damage detection in structures using changes in flexibility", Journal of Sound and Vibration, Vol. 169, pp. 3-17.

[14] Pandey, A. K., Biswas, M. and Samman, M. M. (1991). "Damage detection from changes in curvature mode shapes", Journal of Sound and Vibration, Vol. 145, pp. 321-332.

[15] Patil D.P., Maiti S.K., Experimental verification of a method of detection of multiple cracks in beams based on frequency measurements, Journal of Sound and Vibration 281,(2005), pp.439-451.

[16] Rizos, P. F., Aspragathos, N. and Dimarogonas, A. D. (1990). "Identification of crack location and magnitude in a cantilever beam from the vibration modes", Journal of Sound and Vibration, Vol. 138, pp. 381-388.

[17] Salawu, O. S. (1997). "Detection of structural damage through changes in frequency: A review", Engineering Structures, Vol. 19, pp. 718-723.

[18] Stubb, N. and Kim, J.T. 1996. "Damage Localization in Structures without Baseline Modal Parameter," AIAA Journal,34:1644-1649. 